\title{
Ni phrase ni période? \\ La séquentialité en question dans la diachronie du français
}

\author{
Gilles SIOUFFI \\ Sorbonne Université \\ Gilles.Siouffi@sorbonne-universite.fr \\ https://orcid.org/0000-0003-4426-4967
}

\section{Resumen}

Este artículo plantea, en primer lugar, la pertinencia de las nociones de frase y periodo, para abordar la cuestión general de la secuencialidad discursiva. Confrontamos, para ello, diferentes modelos. A continuación, examinamos la variabilidad diacrónica de los usos, y presentamos los diferentes parámetros de orden externo que han de ser tenidos en cuenta para abordar la cuestión de la secuencialidad de un texto. Finalmente, ilustramos la problemática con tres ejemplos de los siglos XVII y XVIII. Concluimos sugiriendo que la modelización gramatical y las prácticas de puntuación son dos maneras «especificadoras» de ver una cuestión constantemente renovada por la diacronía de las prácticas de escritura.

Palabras clave: frase, período, secuencialidad, discurso en diacronía, cultura escrita.

\section{Résumé}

Cet article interroge la pertinence des notions de phrase et de période pour aborder la question générale de la séquentialité discursive. Il confronte tout d'abord différents modèles. Puis, il examine la variabilité diachronique des usages et présente les différents paramètres d'ordre externe qu'il est nécessaire de prendre en compte avant d'aborder la question de la séquentialité dans un texte. Enfin, il illustre la problématique par trois exemples empruntés aux $\mathrm{XVII}^{\mathrm{e}}$ et XVIII ${ }^{\mathrm{e}}$ siècles. Il conclut en suggérant que la modélisation grammaticale et les pratiques de ponctuation sont deux manières spécifiantes de voir une question que la diachronie des pratiques d'écriture renouvelle sans cesse.

Mots-clés : phrase, période, séquentialité, discours en diachronie, littéracie.

\section{Abstract}

This paper questions the relevance of the notions of sentence and period to address the general question of discursive sequentiality. First of all, it confronts different models. Then, it examines the diachronic variability of uses and presents the various external parameters that

\footnotetext{
*Artículo recibido el 15/09/2020, aceptado el 30/03/2021.
} 
need to be taken into account before addressing the question of sequentiality in a text. Finally, it illustrates the problem with three examples taken from the seventeenth and eighteenth centuries. It concludes by suggesting that grammatical modeling and punctuation practices are two specifying ways of looking at an issue that the diachrony of writing practices constantly renews.

Keywords: sentence, period, sequentiality, discourse in diachrony, literacy.

\section{Introduction}

Phrase et période sont deux termes présents dans le métalangage et la manière de voir le discours des XVII ${ }^{\mathrm{e}}$ et XVIII ${ }^{\mathrm{e}}$ siècles, avec des sens bien particuliers et parfois assez différents les uns des autres (voir la synthèse de Berrendonner 2017), le terme phrase ayant notamment fait l'objet d'une inflexion historique bien connue (voir Seguin, 1993 et Raby, 2018, entre autres), et ils se retrouvent mobilisés dans le modèle théorique récent du Groupe de Fribourg (2012). Cette co-présence ne manque pas de faire question. La période, notamment, est-elle une manière de se représenter le discours typique des XVII e et XVIII siècles, ou peut-on en faire un outil d'analyse " transchronique " qui pourrait être appliqué à n'importe quelle production discursive ? La manière de voir qu'elle introduit est-elle compatible avec celle que suppose la notion de phrase telle qu'elle s'est installée en grammaire depuis la fin du XVIII ${ }^{e}$ siècle ? Ces deux unités de rang 2, pour faire simple, coexistent-elles ou sont-elles deux manières différentes de voir la segmentation d'un texte?

Le fait est que la pertinence de la notion de phrase dans l'approche de certains usages a fait l'objet d'une remise en cause dans les dernières décennies. On pourra citer naturellement en premier lieu les travaux sur l'oral (voir Blanche-Benveniste, 1997 et Morel et Danon-Boileau, 1998, entre autres). Il convient également de citer le travail sur l'écrit des peu lettrés ou sur des corpus écrits ne relevant pas des variétés hautes ou standardisées (voir Ernst, 2003 et Steuckardt, 2015, pour des travaux abordant spécifiquement la problématique). Le programme "Phrase et période en diachronie " du GEHLF (Groupe d'Étude en Histoire de la Langue Française) est d'ailleurs apparu comme un prolongement naturel d'un programme sur les peu lettrés qui avait immédiatement précédé. On pourra également citer les recherches menées actuellement sur la ponctuation dans une perspective historique, qui témoignent d'un souci de revenir plus précisément à la matérialité textuelle et d'en comprendre la logique (voir Dauvois et Dürrenmatt, 2011 : Dürrenmatt, 2011 : Lavrentiev, 2009, Llamas Pombo, 2008 ; Raby, 2016 ; Serça, 2012, entre autres). S'agissant du contemporain, l'apparition de nouveaux usages médiés a amené à porter un nouveau regard sur la frontière oral/écrit et à poser de nouvelles questions aux périodes plus anciennes, comme la prise en compte de ce qu'on appelle aujourd'hui médialité (voir Bruhn, 2016, Siouffi, 2019c). 
Les recherches historiques sur la construction de la grammaire scolaire (voir Chervel, 2008), et les recherches empiriques sur l'usage de la ponctuation et sur la segmentation en contexte scolaire (voir Chanquoy et Fayol, 1995), ont mis en évidence le poids des représentations scolaires. On a pu montrer (Elalouf, 2014), que malgré les difficultés que présente le modèle de la " phrase de base » dans l'analyse de certaines productions comme une longue période de Rousseau, la résistance était grande chez les enseignants à y renoncer. "La phrase est considérée comme la structure fondamentale, à partir de laquelle les autres structures peuvent être définies ", indique d'ailleurs dès ses premières pages le nouveau manuel de terminologie grammaticale proposé aux enseignants par le Ministère français de l'éducation nationale pour la rentrée 2020 (Ministère de l'éducation nationale, $2020: 3$ ). De façon générale, la tendance actuelle en histoire de la langue est de prendre davantage en compte l'interaction entre usages et conscience linguistique, que celle-ci se trouve manifestée dans les descriptions métalinguistiques savantes ou les discours profanes (voir Colombat, Raby, Combettes et Siouffi, 2018).

À la différence du " mot ", la " phrase " (le mot étant placé ici entre guillemets car il peut correspondre à plusieurs concepts différents) ne présente pas de physionomie immédiatement contrainte. Sa réalisation est soumise aux conditions du discours. Or ces conditions changent. La diachronie est un aspect de ces conditions de changement du discours. Peut-on aborder la "phrase " de la même manière dans une chanson de geste du Moyen Âge, un document administratif du XVII siècle, un roman du XVIII ${ }^{e}$ siècle, une lettre de soldat de la Première Guerre mondiale, ou un tweet? De fait, la " phrase " semble poser en termes singuliers ce qu'on peut appeler " diachronie ». Peuton la voir comme un objet qui la traverserait ? Est-elle le reflet d'un modèle ? Faut-il plutôt parler de manière de voir ? C'est cette ambiguïté qui fait toute la richesse de la problématique.

Le point de départ des réflexions que nous présentons ici est à situer dans une série de travaux menés dans le cadre du GEHLF, (voir Baudry et Caron, 1998 ; Combettes, 2003). Récemment, le GEHLF a consacré un séminaire de deux ans (20152107) à la question " Phrase et période en diachronie ", en essayant de confronter divers modèles théoriques et en examinant la question sur tout l'empan de la diachronie. Un certain nombre de ces travaux sont parus, notamment ceux portant sur les XVI ${ }^{\text {e}}$ XVIII ${ }^{\mathrm{e}}$ siècles (Siouffi, 2019b), et d'autres sur toute l'histoire du français (Siouffi, 2020). Ce qui suit doit beaucoup aux interventions réalisées dans le cadre de ce séminaire. L'objectif est ici de faire écho à quelques-unes des réflexions qui ont émergé, ainsi que d'expliciter et de déplier quelques questions plus larges qu'elles ont fait surgir.

Il s'agira d'abord de confronter la question des modèles et celle des usages. Après plusieurs décennies où on s'est beaucoup concentré sur les notions d'un point de vue de théorie linguistique, on observe en effet aujourd'hui un retour vers les usages qui correspond aux modifications des méthodes de ce qu'on peut appeler de façon large 
"philologie ", et à l'émergence de la sociolinguistique historique. Si l'on part du plan du modèle, nous montrerons comment les notions de phrase et de période, notamment telle que la définit le Groupe de Fribourg (2012) sont deux manières d'aborder le problème général de la séquentialité.

Dans un deuxième temps, en partant des usages, on se demandera quels sont les paramètres, notamment externes, dont on doit tenir compte pour envisager dans la diachronie la question de la séquentialité.

Enfin, on illustrera ce problème général de la séquentialité (comment commencer une " phrase » ? comment la finir ?) par trois études de cas empruntés aux XVII et XVIII" siècles.

\section{Si l'on part du modèle}

\subsection{Que faire de la «phrase" ?}

Que faire de la " phrase » si on l'envisage comme notion ? Loin de nous l'idée de résumer en quelques lignes les débats auxquels une immense littérature a été consacrée. Toutefois, en s'inspirant de Le Goffic (2008 et 2011), on peut formaliser quatre attitudes possibles :

(a) On peut la postuler, la poser comme un a priori, la " décider ». C'est ce que fait d'une certaine manière la grammaire scolaire. La plupart du temps, dans cette perspective, on assimile la phrase soit à l'énoncé, soit à la proposition logique, ou on cherche à la définir par ses limites (en s'aidant beaucoup de la ponctuation et du sens).

(b) On peut en construire le modèle dans une perspective ascendante : en partant des constituants, et notamment du noyau que constitue le verbe conjugué. Ce qu'on appelle phrase devient le lieu de fonctions, d'accords, etc. - un lieu d'analyse syntaxique. Ainsi Le Goffic définit-il la phrase en rattachant au noyau les éléments qui en dépendent et les éléments qui portent sur la phrase elle-même (réflexivité intégrée) ; ou le Groupe d'Aix construit-il cette perspective ascendante au moyen de deux modules : microsyntaxique et macrosyntaxique (Blanche-Benveniste, 1997 ; Benzitoun et Sabio, 2010). La question qui se pose ensuite est de savoir jusqu'où aller, au-delà de la phrase, si on veut maintenir la perspective syntaxique (Charolles et al., 2002).

(c) On peut l'envisager dans une perspective descendante, à partir de l'unité de rang supérieur. La plupart du temps, cette unité est le texte (voir Combettes, 1983, Adam, 2003), ce qui suppose qu'une phrase s'insère dans une organisation plus large dans laquelle ses éléments jouent aussi un rôle. Cette perspective met bien en évidence la différence de physionomie syntaxique qu'il peut y avoir entre des phrases isolées et des phrases prenant place dans un texte.

(d) On peut enfin la nier et considérer que le discours fonctionne (dans certains cas) sur autre chose que des phrases. 
Pour ceux qui acceptent d'en reprendre le modèle, en tout cas, la phrase apparaîtra facilement comme un " miroir à deux faces ", qui d'un côté regarde la "syntaxe ", définie comme module d'organisation des constituants selon des règles linguistiques, de l'autre le texte, gouverné par des procédures d'organisation différentes, moins réglées, fondées sur la segmentation et la séquentialité.

Ce double caractère peut inviter à considérer, comme le fait Le Goffic, que le discours s'organise fondamentalement en deux modules, un module textuel et un module syntaxique, qui se superposent. Un observatoire de cette superposition est par exemple la ponctuation : parfois, la ponctuation a clairement une fonction syntaxique, parfois, elle joue un rôle dans le module textuel.

Une autre option, celle du Groupe de Fribourg (2012), est de formaliser une distinction entre deux échelles : clause et période. Selon le Groupe de Fribourg, la clause, ou énonciation, comprend une construction verbale et des compléments régis, et constitue l'unité maximale de la micro-syntaxe. La période, de son côté, est une unité supérieure, multiclausale, qui peut inclure des parenthèses, des boucles énonciatives, des faits de discours rapporté, etc. Elle ne relève pas de la microsyntaxe, mais constitue un agencement, une configuration. De la sorte, la "clause " pourrait présenter une partie des fonctions attribuées par la grammaire à la " phrase ».

\subsection{Un modèle de séquentialité}

L'introduction de modèles présentant des échelles différentes, ou des superpositions de modules, révèle à vrai dire que notre approche de la textualité reste très marquée par une idée de la " phrase " qui rêve de voir y coïncider l'unité syntaxique, l'unité sémantique et l'unité énonciative. On le sait, la notion de phrase est installée sous ce nom dans le paysage de la description linguistique (français) depuis Domergue (fin $18^{\mathrm{e}}$ ) et surtout depuis son exploitation par l'institution scolaire au $19^{\mathrm{e}}$, dans un double mouvement de grammatisation et de syntactisation de notre regard, de sorte que beaucoup des modèles linguistiques d'aujourd'hui sont basés sur la syntaxe. On peut se poser la question de savoir si ce processus n'induit pas un regard partiel et biaisé sur de nombreuses productions discursives.

$\mathrm{Si}, \mathrm{du}$ point de vue du discours, on peut juger que la réflexion sur la phrase relève de la problématique plus générale de la segmentation et de la séquentialité, il n'est pas sûr que la forme de séquentialité appelée aujourd'hui " phrase » soit la seule, ni toujours la plus opérante. On constate en effet que beaucoup de segmentations ou d'associations larges ne sont pas des "périodes" au sens classique, mais plutôt des assemblages, qui n'ont pas de configuration particulière. Malgré tout ces assemblages font sens, pas tant au niveau sémantique qu'au niveau de l'organisation discursive.

On l'a dit, ce sont les travaux sur l'oral qui ont mis en évidence d'autres principes d'organisation (voir Hirsch et Dodane, 2018 et Lacheret et Victorri, 2002). Soit la phrase n'est pas considérée comme pertinente, soit elle n'apparaît pas comme la seule 
manière de voir la segmentation. N'en serait-il pas de même pour l'écrit ? Cette mise en question de la phrase comme unité fondamentale de la segmentation parle de fait à un diachronicien. L'examen des usages, notamment ceux qui précèdent le XVIII siècle, invite à rechercher d'autres principes d'organisation. Les textes manuscrits des peu lettrés le montrent, bien sûr, mais également les pratiques d'impression, et l'histoire de la ponctuation.

Fondamentalement, on peut considérer que la conception d'un double module (syntaxique et textuel) du discours et la modélisation en clause / période posent - différemment, et chacune à leur manière - la question essentielle de la séquentialité du discours. Le terme séquentialité décrit la séquence linguistique d'unités agencées linéairement de manière dynamique, comme un ensemble émergent, en devenir, pouvant être ensuite soumis à des interprétations sémantiques comme formelles, et à des propositions de réception, par exemple en terme de " phrases ». Selon Apothéloz (2007: 42), " la question de la séquentialité soulève celle de l'articulation entre grammaire et activité, ou plus précisément : entre le déroulement temporel des structures linguistiques et l'enchaînement séquentiel des actions de discours ».

De fait, on ne voit pas comment, du point de vue de la production, la séquentialité peut s'appréhender dans une perspective ascendante (depuis les unités). Il y a là presque une contradiction dans les termes. En revanche, du point de vue de la réception, elle peut s'envisager selon les deux perspectives, ascendante ou descendante (depuis l'ensemble), ce qui légitime le double regard, syntaxique et discursif.

\subsection{Des modèles encombrants?}

Il est possible que la "phrase ", entendue dans une perspective ascendante comme agrégation d'éléments autour d'un noyau, soit une unité spontanée, intuitive, ou observable de la séquentialité en action. Mais il y aurait peut-être un risque à la confondre d'emblée avec la "phrase " qui résulte de notre analyse syntaxique. C'est pourquoi nous nous retrouverons pour notre part assez bien dans ces propos de Guillaume, qui, comme on lui reprochait de ne pas suffisamment définir la phrase, répondait :

La définition de la phrase en tant qu'unité d'effet de discours a le mérite, par son accord avec le sentiment du locuteur [...], de ne pas prêter le flanc à la controverse. Elle est quelque chose qui s'admet naturellement, étant entendu que la définition ainsi formulée ne vaut que dans le cadre de la relativité réciproque des deux entités de langage, phrase et mot, et n'a pas la prétention de donner de la phrase une définition qui en embrasserait tous les aspects (Leçon du 27 novembre 1941, in Guillaume, 2005 : 40). 
Le sentiment des locuteurs (variable bien évidemment en diachronie, mais aussi selon un assez grand nombre de paramètres, dont les paramètres discursifs) joue vraisemblablement un rôle décisif dans la détermination de ce qu'on peut accepter comme "phrases ».

Du point de vue des usages, le problème de la séquentialité se divise en deux problèmes distincts : un problème syntaxique, et un problème de segmentation textuelle. Ces deux problèmes doivent être envisagés différemment selon qu'on se place du point de vue de la production ou du point de vue de la réception. Comme beaucoup de chercheurs l'ont montré, la syntaxe et la segmentation sont des exigences de production qui donnent lieu à une programmation - programmation syntaxique (surtout) comme, à un moindre degré, programmation de segmentation textuelle. Du côté de la réception, la syntaxe et la segmentation sont aussi des exigences, exigences qui peuvent donner lieu à une analyse, dans le cas de la syntaxe, et à une lecture, dans le cas de la segmentation - la lecture étant ici à comprendre comme une résolution textuelle. Il résulte de ces différences de points de vue et de ces superpositions de procédures des possibilités de conflits (voir, par exemple, Gautier, 2010, sur l'interprétation du point). C'est la raison pour laquelle, si l'on part des usages et non des modèles, les conditions de production et de réception doivent être interrogées.

\section{Si l'on part des usages}

Nous nous proposons à présent de dire quelques mots des principaux paramètres qu'il nous semble nécessaire de prendre en compte pour évaluer correctement les conditions de production et de réception.

\subsection{L'intégration à la textualité}

Dans les dernières décennies, le retour aux usages a sans doute été en partie motivé par le fait qu'une partie du travail linguistique qui avait précédé s'était effectué sur des phrases sorties de leur contexte, voire des phrases forgées. Le retour aux usages a d'abord permis de mettre en évidence la textualité. Voici trois citations de Weinrich (1989 : 19-20) qui exemplifient cette position :

Une grammaire qui se présente comme une grammaire textuelle ne peut qu'être entièrement conçue à partir de textes (oraux ou écrits), puisque son objectif ultime est de conduire à manier la langue dans des textes. [...] Nous appelons texte l'énoncé linéaire qui est compris entre deux interruptions remarquables de la communication et qui va des organes de la parole ou de l'écriture de l'émetteur aux organes de l'audition ou de la vue du récepteur. [...] Il s'ensuit qu'un objectif prioritaire de la description linguistique est de saisir la textualité d'un texte. Nous entendrons par là la cohérence particulière qui fait qu'un texte est un texte. 
Au final la textualité ainsi comprise devient un paramètre, au sens où l'analyse de la «phrase en usage » implique qu'on prenne en compte la textualité dans laquelle cette phrase intervient.

Cette textualité dépend au premier chef du genre de discours dans lequel la phrase est prise. On peut se demander de ce point de vue s'il n'existe pas ce qu'on pourrait appeler des " phrases de genre ", dans l'oral comme dans l'écrit (l'interview, la lettre, le sermon...). Par ailleurs, on sait qu'il existe dans l'histoire de la culture une longue tradition de textes formés de phrases non insérées (proverbes, slogans, maximes...). Ce caractère détaché n'implique pas qu'on n'ait pas affaire à une textualité.

\subsection{L'interaction}

Qui dit usage dit interaction. Il s'agit alors de savoir dans quelle mesure le discours est adressé. Lorsque Weinrich (1989 : 20) construit l'idée d'une grammaire « textuelle ", il élabore une grammaire de la réception au sens où, pour lui, " seuls les messages qui atteignent leur destination ont leur pleine signification communicative ». Il part ainsi, pour analyser la physionomie des énoncés, de la supposition dans tout texte d'une instruction du type "cher auditeur [ou lecteur], si tu veux que ce texte ait un sens pour toi, alors voici comment tu dois te comporter" (Weinrich 1989:21).

On peut néanmoins se demander si la réception d'un texte est nécessairement celle d'un texte qui nous a été adressé (selon un schéma de la communication pris au pied de la lettre). A la vérité, quand on examine le corpus des textes écrits sur lesquels la discipline de la grammaire a développé ses méthodes et ses concepts, on s'aperçoit qu'il s'agit la plupart du temps de textes qui ne sont pas vraiment adressés. Sans aller jusqu'aux monologues, soliloques, le texte littéraire, sur lequel se fondent une grande partie, à la fois de la littéracie, et de la description grammaticale, n'est pas vraiment " adressé " dans ce sens. Il n'en reste pas moins que ce critère de l' "adressage ", si l'on peut dire ainsi, reste fondamental dans l'approche générale des discours et spécifiquement de la séquentialité.

\subsection{La médialité}

La question de la médialité est corrélative de celle de l'interaction. Par médialité on entendra la médiation communicationnelle ou pragmatique introduite par le médium. Nous suivrons l'analyse de Bruhn (2016), pour qui il est plus difficile d'isoler ce qu'on peut appeler médium que de parler en termes de processus, ce qu'implique le nouveau terme de médialité (mediality). La prise en compte de la médialité conduit à réviser profondément notre regard sur ce qu'on a longtemps considéré comme l'écrit pour mettre en avant la dimension d'écriture (en anglais Writing).

La médialité comme processus induit des contraintes et des affordances. Les contraintes sont les limitations auxquelles va se heurter la communication : limitations 
en termes d'espace lorsqu'il s'agit d'écrire une carte postale ou un tweet, par exemple. Les affordances, si l'on suit Kavanagh et al. (2016), peuvent être de plusieurs types. Dans cet ensemble, on peut ranger les affordances médiales, dont la ponctuation, par exemple, qui dépend des conditions de scripturalité, est une conséquence. De fait, la notion de médialité, souvent sollicitée pour rendre compte des écritures numériques actuelles, pourrait sans doute être davantage convoquée à propos des époques anciennes, tant en ce qui concerne la différence entre manuscrits et imprimés (à l'époque médiévale notamment) que dans le domaine plus restreint des imprimés de l'époque moderne.

Du point de vue de la production, ce qu'on peut appeler " phrase » se trouve au centre d'une interaction dialectique entre énonciation et médialité. La différence entre les canaux de l'écrit et de l'oral est centrale dans cet enjeu. Si un programme communicationnel similaire peut être envisagé, la différence des contraintes et des affordances est flagrante. Du point de vue de la réception, par ailleurs, l'existence de " phrases " est conditionnée par un besoin de segmentation qui rend difficilement recevables, par exemple, des textes écrits proposés sans aucune ponctuation ou des suites ininterrompues de discours non segmentées par des indications prosodiques. Au total, s'il est un plan de la réalisation linguistique dans lequel la médialité joue, c'est bien la phrase.

\subsection{La littéracie et les cadres de pensée}

Par littéracie, on peut entendre, non seulement, classiquement, la capacité à lire et à écrire, mais aussi ce qu'on appelle parfois la "syntactisation ", ensemble de pratiques grammaticales, de ponctuation et de segmentation résultant d'un apprentissage (voir Chanquoy et Fayol, 1995; Roubaud, 2016). La littéracie crée des " cadres de pensée " au sens où elle s'appuie sur un métalangage explicite comme, dans l'histoire, les termes proposition, phrase, période..., recevant chacun, et selon les grammaires, leurs définitions, mais aussi sur une certaine façon de court-circuiter la description savante au profit de manières de voir assez simples permettant de couper court aux discussions. Lorsqu'un manuel du XIX siècle propose : "Faites observer aussi que vous commencez chaque phrase par une majuscule et que vous la terminez par un point final. Le mot phrase s'expliquera de lui-même par l'usage. " (Anon, 1860 : 279), il installe dans le paysage une manière de voir la question qui, si elle ne satisfait certes pas le grammairien, n'est pas dénuée d'impact.

En dépit de ses avantages en termes de compétence, la littéracie engendre des manières simples, pour ne pas dire simplistes, de comprendre les faits de langue. Elle produit aussi une unification notionnelle. En effet, il est préférable, dans le processus de syntactisation, qu'une manière de voir l'emporte sur les autres, et élimine ses concurrentes - ce qui pose d'ailleurs des problèmes dans l'institution scolaire contemporaine ; et elle a ensuite pour conséquence que la notion l'emporte sur les autres manières 
de voir. À partir du moment où l'institution scolaire s'est focalisée (à la fin du XIX siècle) autour de la "phrase ", ainsi, on a vu leur pertinence et leur efficience retirées aux autres modèles, comme celui de la période dans son sens classique (voir Gautier, 2006). L'avantage cognitif de cette simplification est alors de décomposer notre perception de la langue et du discours en deux zones : le typique et l'atypique ; et de comprendre l'atypique par le typique. Certes, la description linguistique, dans son sens le plus ambitieux, peut être vue comme une manière de chercher à comprendre l'atypique sans passer par la référence obligée au typique, mais on constate aussi qu'elle en revient souvent, subissant sans doute l'influence des cadres de pensée d'une époque, à conforter la description du typique par l'atypique.

Au total, on pourra considérer que la production de la phrase en discours est l'effet d'une programmation qui est confrontée à un grand nombre de paramètres, contraintes et affordances diverses qui ne vont d'ailleurs pas toujours dans le même sens. Outre les contraintes cognitives liées à la réalisation de la syntaxe, on doit prendre en compte les paramètres d'intégration textuelle, d'interaction, les contraintes et les affordances médiales, et l'arrière-plan décisif que représente la littéracie. C’est ainsi que, si on veut partir des usages et considérer la " phrase " en diachronie, on devra accepter que la question de la phrase n'est pas seulement une question linguistique. Ce n'est pas sur une base « linguistique » que Llamas-Pombo (2017) a pu montrer que la ponctuation des chartes médiévales ne se laissait pas décrire de façon pertinente en termes de " phrase " : bien plutôt, une étude attentive de la textualité, des paramètres énonciatifs, et de la scripturalité ont permis de mettre en évidence d'autres modes de fonctionnement.

C'est un travail similaire que nous proposons à présent d'expérimenter à présent à partir de textes des XVII et ${ }^{\mathrm{e}}$ XVIII ${ }^{\mathrm{e}}$ siècles.

\section{Phrase ou période ? quelques observatoires (XVII $-\mathrm{XVIII}^{\mathrm{e}}$ siècles)}

Nous allons à présent procéder à trois études de cas empruntés aux XVII et XVIII ${ }^{e}$ siècles afin d'étudier les problèmes que posent la segmentation et l'association de segments, en nous concentrant sur le problème des segments qui peuvent être perçus comme possédant toutes les caractéristiques d'une " phrase ", ou être autonomes. Rappelons que les outils disponibles en production comme en réception pour résoudre ces problèmes sont :

- des outils linguistiques grammaticalisés, comme les connecteurs (voir BadiouMonferran, 2000), et non grammaticalisés, comme certains noms thématisants, dont l'exploration est sans doute à approfondir ;

- des outils graphiques de ponctuation ;

- des outils visuels comme les alinéas (voir Arabyan, 2018), à distinguer de notre point de vue des outils de ponctuation; 
- dans le cadre de l'oral, des outils prosodiques, comme des pauses, des reprises de souffle, l'intonation, etc.

\subsection{Le cadre de pensée}

Au début du XVII ${ }^{e}$ siècle, la situation a été décrite en ces termes par Jean-Pierre Seguin :

Une situation polylectale latente fait coexister d'abord trois modèles : celui, ancien, d'un discours continu ; celui de la période, avec référence orale prioritaire et prédominance oratoire; et celui d'un découpage en entités énonciatives qui cherchent une clôture moins vaste, préparant la pratique et l'idée de la phrase actuelle (Seguin, in Combettes, 2003 : 69).

Le modèle de la période est loin d'être le seul à s'appliquer à la phrase longue, dans la mesure où existe de manière forte un modèle de " phrases liées » (voir Mounier, in Siouffi, 2019b).

Les outils linguistiques font l'objet de tentatives de réglages explicites, et commencent à dessiner ce que nous nommé un "cadre de pensée ». Vaugelas essaye par exemple de régler l'usage d'outils grammaticalisés dans le rôle d'association d'unités comprises comme des "périodes", acceptant de ce fait et, mais non plus qui, outil traditionnel de l'association en latin et au XVIe siècle :

$\mathrm{Au}$ reste, on peut fort bien commencer une periode par la conjonction $e t$, je dis mesme lors qu'il y a un point, qui ferme la periode precedente. Je n'en rapporteray point d'exemples, parce que tous nos bons Autheurs en sont pleins. Nous avons si peu de liaison pour les periodes, qu'il ne faut pas encore nous oster celle-cy (Vaugelas, 2009 : 637).

Nous avons quelques Escrivains, qui après avoir fait une longue periode sans avoir achevé ce qu'ils veulent dire, se sont avisez d'un mauvais expedient, pour faire d'un costé que la periode ne passe pas les bornes, et que d'autre-part ils y puissent ajouster ce qui luy manque. Voicy comme ils font. Quand le sens est complet, ils mettent un point, et puis commencent une autre periode par le relatif qui. [...] Je dis [...], que de faire un point devant ce qui, et de commencer une autre periode par ce mot, est un fort mauvais remede, dont nous n'usons jamais en nostre langue. Il est vray que les Latins se donnent ordinairement cette licence, et c'est à leur imitation que les Escrivains dont je parle, le font : mais nous sommes plus exacts en notre langue, et en nostre stile, que les Latins, ny que toutes les Nations, dont nous lisons les escrits [...] (Vaugelas, $2009: 259-260$ ). 
Du côté de la ponctuation, on observe également des tentatives de réglage. À la fin du XVI ${ }^{\mathrm{e}}$ siècle, Jean Bosquet $(2005$ : 131) formule la fonction démarcative du point : «Le poinct clost et finist non seulement le sens de la clause, ou devis; mais tout le parler et discours : De sorte, que sy après iceluy suit quelque chose, c'est autre matière, quy arriere se commence ».

Au milieu du XVII ${ }^{e}$, Charles Sorel va bien loin, proposant un réglage permettant de différencier le point virgule et le deux-points dans l'association de ce qu'il appelle les «demi-périodes":

On se doit servir des deux points à la fin d'une demye période, lors que celle qui suit commence par quelque Conjonction, ou bien lors que le Sens est entierement dependant des clauses précédentes, \& tiré en leur consequence. Pour le poinct à queüe il sert à la fin d'une demye période dont le Sens est presque complet, \& le poinct est toujours mis à la fin d'une periode entiere (Sorel, $1688: 115$ ).

En faisant cette proposition de distinction sur critère sémantique, il entre visiblement dans la "vraie question ", qui est celle de la segmentation intermédiaire, et qui est la question sur laquelle, du point de vue de la séquentialité, au terme de plus d'un siècle d'élaboration théorique, avec Domergue, la notion de phrase apportera quelque chose de supplémentaire par rapport à la notion de période.

Si l'on compare les trois définitions que donnent de période les trois grands dictionnaires monolingues de la fin du XVII ${ }^{e}$ siècle, en effet :

PERIODE. [...] en termes de Grammaire, est une petite estendüe de discours qui contient un sens parfait, \& qui ne doit pas estre plus longue que la portée ordinaire de l'haleine.(Furetière, Dictionnaire universel, 1690).

Periode : La periode composée est une sorte d'élocution achevée $\&$ parfaite pour le sens, qui a des parties distinguées \& qui est facile à prononcer tout d'une haleine. (Richelet, Dictionnaire françois, 1680).

Periode : s. f. Portion d'un discours qui consiste en un certain arrangement de paroles, \& qui estant composée de plusieurs membres, renferme un sens complet. (Dictionnaire de l'Académie, 1694).

on constatera que, si divers critères sont pris en compte (sémantiques, syntaxiques, prosodiques), seule l'Académie évoque la question des membres.

En fait, la définition rhétorique de la période s'offrait comme une résolution par l'esthétique (longueur des membres, etc.) du problème de la séquentialité. Pour autant, elle ne permet pas de mettre un nom unique sur toutes les formes d'association 
de clauses destinées à former des unités longues. Au début du XVIII siècle, le grammairien Buffier repère bien le problème :

Les périodes du style coupé consistent en plusieurs phrases ou expressions, qui souvent prises chacune en particulier, semblent faire un sens complet ; $\&$ pourtant ce ne sont que des phrases ou des propositions particulières subordonnées à une proposition principale, dont elles marquent les diverses circonstances. Quelques-uns peut-être ne voudront pas appeler périodes, cet amas de diverses phrases qui sont sans liaison grammaticale. A eux permis ; nous faisons profession de ne jamais disputer sur les mots. (Buffier, 1709 : 430).

Il relève que ce qui est qualifié depuis peu ${ }^{1}$, d'un point de vue rhétorique, de " style coupé » ne consiste ni plus ni moins qu'en la tendance à associer dans des unités longues des segments pouvant être autonomes. Ce faisant, il met le doigt sur la vraie difficulté de la séquentialité telle qu'elle est vue par la tradition rhétorique. S’appuyer sur la notion de période entendue dans son sens classique ne revient en effet à ranger sous ce nom, dans la continuité de la tradition antique, qu'un certain type de phrases longues, notamment celles qui présentent une organisation rythmique particulière. Échappent à la description un grand nombre de séquences longues dans lesquelles des segments détachables sont souplement reliés les uns aux autres, accumulés, ou laissés dans le sentiment d'une indépendance possible.

Porter le regard sur trois moments de la diachronie des XVII ${ }^{\mathrm{e}}$ et XVIII ${ }^{\mathrm{e}}$ siècles, et sur des types de pratiques différents, permettra peut-être de mieux identifier quelques-unes des questions précises qui traversent cette problématique générale de la séquentialité.

\subsection{Les Lettres meslées de Tristan L'Hermite (1642)}

La physionomie qu'offrent les Lettres meslées de Tristan L'Hermite parues chez Augustin Courbé en 1642 (voir Siouffi, 2019a) est le résultat des choix d'un imprimeur, qui mobilise une ponctuation remplissant encore, au début du XVII siècle, plusieurs fonctions : une fonction syntaxique (comme le montrent les virgules entre sujet et verbe), une fonction rythmique, et une fonction de segmentation du discours. Certes, il y a danger à surinterpréter ces signes non encore soumis à des normes régulatrices ; il n'en demeure pas moins qu'on peut se fonder sur la présence quantitativement importante de certains faits pour formuler des hypothèses.

\footnotetext{
${ }^{1}$ Même si la métaphore de la coupure se rencontre ici et là depuis le XVI ${ }^{\mathrm{e}}$ siècle, la popularisation du syntagme semble dater de sa présence dans le dictionnaire de Furetière de 1690 (voir Lecointe, 2002 : 13-14).
} 


\section{IETTRES}

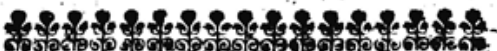

\section{A C LIT YE. Sur fon merite.}

\section{LETTRE XXXI.}

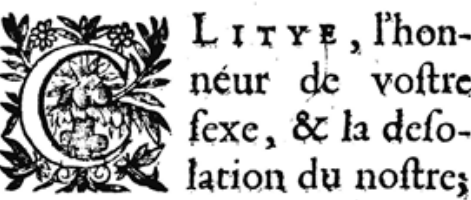

Beauté à qui l'on offre tant $d^{2} i l l u f t r e s$ victimes, \& qui n'en daignez accepter pas vne. Quels magnifiques trophées peut-on efleuer à voftre gloire, qui ne foient au

Fig. 1. Tristan l'Hermite, Lettres meslées, (1642: 182)

Dans ce texte imprimé, la virgule, le point, le deux-points et le point virgule sont donc les quatre outils de ponctuation utilisés pour segmenter et associer. On observe de nombreux cas de juxtaposition de segments autonomes par des virgules, par exemple - formule qui sera critiquée par la grammaire scolaire ultérieure. Il existe aussi des cas d'apostrophes clôturées par des points.

Visiblement, l'apostrophe (un peu longue, il est vrai), est perçue comme une clause pouvant être comprise comme autonome. Les subordonnées à gauche du prédicat central, également, sont souvent segmentées par des points, selon une pratique de l'époque, qu'on trouvait déjà chez Montaigne.

Dans le passage suivant, trois outils différents sont employés devant des complétives associées : 


AMOVREVSES. In
me doiue iamais donner le
temps de vous dire quatre
paroles. Si ie pouuois treu-
uer locafion de vous parler
en liberté, vous aprendriez
de mapaffion, beaucoup d'a-
greables nouuelles dont vo-
ftre Miroir vous a defia dé-
couuert vne partie. Vous
fçauriez que iadore le plus
beau de tous les Miracles vi-
fibles; que ie brufle pour des
yeux qui font de la couleur
des Saphirs, mais qui bril-
lent beaucoup plus que sils
eftoient des Diamans: Que
ie fuis charmé du vif éclat,
\& du fon de voix, dvne
H iiij

Fig. 2 et 3. Tristan L'Hermite, Lettres meslées (1642: 119-120)

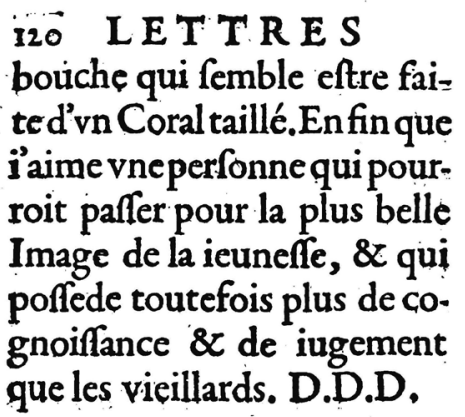

\section{Yoltre, \&c.}

Certes, on pourra toujours voir dans ce type d'exemples la preuve d'une absence de standardisation des pratiques. Nous pensons au contraire que cette variation présente un intérêt, et qu'il est possible de voir un mode de construction discursive original dans cette présentation échelonnée. En l'occurrence, la gradation des signes de ponctuation alliée à l'élargissement volumétrique semble donner une autonomie possible de plus en plus grande aux segments, en faisant peut-être des clauses énonciatives détachables, même si elles sont ramenées à une énonciation principale.

Du point de vue grammatical, on note également la grande fréquence des ajouts après prédicat, ce qui montre que celui-ci ne signe pas véritablement l'identité phrastique dans le sens moderne. On relève ici le rôle essentiel du $E t(\&)$, très fréquent après le deux-points ou le point-virgule (voir Badiou-Monferran et Capin, 2020 : 236, 275, 470, sur le et de relance), les tellement que, ou de sorte que après un point, les comme après deux-points. Tous ces procédés, très récurrents, transforment ce qui serait aujourd'hui considéré comme des ajouts circonstanciels dans une phrase en éléments extra-prédicatifs intervenant en marge du prédicat central et susceptibles de donner naissance à une nouvelle énonciation. À force d'hyperbates et de rebonds, on obtient ainsi un tissu qui n'a plus de raisons de s'interrompre :

Vous seriez bien marrie de respondre mieux à mes Lettres, qu'à ma passion : de sorte que ie puis assurer que ie ne sers pas seulement une personne inexorable; mais encore une femme qui feint d'estre tout ensemble, aveugle, 
sourdre \& paralitique. Et bien, Glicere, c'est une Destinée que ma prudence ne peut changer, il faut que ie vouë toute ma vie à l'ingratitude ; \& que ie ne reçoive iamais pourtant d'amour, de soins \& d'obeissance, que de l'indiference, ou du mepris. Puis que ce m'est une necessité de vous aimer iusqu'au tombeau, ie n'ay qu'à chercher dans mes seules larmes, la consolation de ma douleur : \& à me plaindre autant du mal-heur d'auoir esté blessé de vos yeux, que si i'avois esté frapé du Tonnerre : Mais c'est une chose bien estrange que les Diuinitez tiennent à gloire de foudroyer leurs propres Autels. (Tristan L'Hermite, $1642: 127-128$ ).

L'association des segments produit-elle ici une " phrase " au sens moderne ? une période au sens rhétorique ? C'est bien difficile à dire dans les deux cas. La ponctuation comme les outils linguistiques - autant lexicaux que grammaticaux - construisent une continuité de segment à segment qui rend difficile l'établissement de délimitations autres, qui surplomberaient ce fonctionnement. Le texte est fait de toutes sortes d'appareillages qui sont ensuite combinés au moyen d'outils dont la distribution ne paraît pas toujours répondre à une logique identifiable, et suit des décisions qui, naturellement, ne sont pas apparentes. Association et segmentation sont tellement solidaires, dans ce processus, que c'est l'arrêt qui finit par poser un problème. D'ailleurs, l'ajout après le point, parfois analysé dans la tradition rhétorique sous le nom d'hyperbate, est une caractéristique récurrente de la textualité dans la diachronie.

Observé ici dans un imprimé du milieu du XVII siècle, où il n'occupe d'ailleurs pas toute la place, ce type de segmentation du discours se heurtera bientôt aux propositions de réglages faites par les imprimeurs et à la modification des cadres de pensée. Un changement dans l'usage des connecteurs accompagnera cette évolution à la fin du XVII ${ }^{e}$ siècle (Badiou-Monferran, 2000). L'éventail des segments considérés comme détachables se réduira autour des segments prédicatifs.

Voyons malgré tout ce qu'il en est dans un texte au statut particulier : le sermon sur la mort de Bossuet (dit en 1662, publié en 1767).

\subsection{Les sermons de Bossuet}

Les sermons de Bossuet offrent le cas de textes qui ont fait des aller-retours entre les canaux de l'oral et de l'écrit. On suppose généralement que Bossuet jetait par écrit un canevas de ses sermons, qu'il produisait à partir de ce canevas une performance orale, puis qu'il retranscrivait cette performance sous la forme d'un manuscrit plus élaboré. Toutefois, lorsque nous ne possédons qu'un manuscrit autographe élaboré, il nous est difficile d'affirmer si celui-ci a précédé ou suivi la performance orale. Un seul de ces sermons fut publié du vivant de l'auteur. Ce n'est que plus d'un demi-siècle après la mort de Bossuet que le bénédictin René Déforis, en 1772 (Bossuet, 1772), publia un choix de ces sermons, avant l'édition Lebarcq, plus tard revue par Urbain et Levesque (Bossuet, 1914-1921). 
Comparer les divers états d'un même passage se révèle instructif quant aux pratiques de segmentation.

Selon une pratique courante à l'époque, le manuscrit (BnF, fr. 12822) n'est que peu ponctué. À cela s'ajoute le fait que le genre du sermon suppose une médiation orale, et il est raisonnable de considérer que la syntaxe de la version écrite dont nous disposons a pu être influencée par le paramètre de cette réalisation orale. Enfin, on peut considérer que l'interaction (dans le domaine de la distance communicative) est ici un paramètre pragmatique fondamental.

Si l'on retranscrit le manuscrit, en tout cas, on s'aperçoit vite que le texte n'est pas structuré en "phrases " au sens moderne, et peut-être pas non plus en "périodes " au sens classique. On a plutôt affaire à un projet énonciatif large, qui déborde clairement l'unité de ce qu'on pourrait entendre aujourd'hui par " phrase » et qui se structure ensuite au fur et à mesure au moyen de connecteurs (à l'usage parfois délicat à cerner), et de liens sémantiques entre les segments ou entre les mots.

Considérons l'extrait suivant. Le segment est assez long, mais on ne peut réellement comprendre comment le discours se structure qu'en le saisissant dans cet empan:

Quest-ce que cent ans, quest-ce que mil ans puisqu'un seul moment les efface. Multipliez vos iours comme les cerfs que la fable ou l'histoire de la nature fait vivre durant tant de siecles durez autant que ces grands chenes sous lesquels nos ancestres se sont reposez et qui donneront encore de l'ombre a notre posterité, entassez dans cet espace qui paroit immense, honneurs, richesses, plaisirs, que vous profitera cet amas puisque le dernier soufle de la mort tout faible, tout languissant abatra tout a coup cette vaine pompe avec la mesme facilité qu'un chateau de cartes vain amusement des enfans ? et que vous servira d'avoir tant ecrit dans ce livre, d'en avoir rempli toutes les pages de beaux caracteres puisqu'enfin une seule rature doit tout effacer. Encore une rature laisseroit-elle quelques traces du moins d'elle mesme au lieu que ce dernier moment qui effacera d'un seul trait toute votre vie s'ira perdre luy mesme avec tout le reste dans ce grand goufre du neant. Il n'y aura plus sur la terre aucuns vestiges de ce que nous sommes; la chair changera de nature ; le cors prendra un autre nom ; mesme celuy de cadavre dit Tertullien ne luy demeurera pas long temps : il deviendra un ie ne sais quoy qui n'a plus de nom dans aucune langue tant il est vray que tout meurt en luy jusqua ces termes funebres par lesquels on exprimoit ses malheureux restes. (Bossuet, Ms. BnF, fr. : f. 361v.).

On voit bien qu'on assiste ici à une accumulation de courtes unités dont toute la difficulté est de dire si elles sont liées les unes aux autres ou autonomes. Nous avons surligné en gras les passages où les interventions des éditeurs vont révéler des interprétations différentes de cette séquentialité si particulière. Du début, Déforis va faire, par exemple : "Qu'est-ce que cent ans? qu'est-ce que mille ans, puisqu'un seul moment 
les efface ? ", ce qui dénote un alignement du sens de la phrase sur le noyau prédicatif qui suit l'élaboration grammaticale alors en cours, alors que Urbain et Levesque vont proposer : "Qu'est-ce que cent ans, qu'est-ce que mille ans, puisqu'un seul moment les efface ?", ce qui donne une phrase amplifiée et correspond plutôt à l'idée que l'époque se fait, sinon de la période (un peu limitée, ici), au moins de l'éloquence.

À partir de "Multipliez... ", on entre dans la logique d'une vaste énonciation interrogative (dédoublée), suivie bientôt d'une série de segments assertifs qui paraissent juxtaposés. Du passage surligné, Déforis fait :

Encore une rature laisseroit-elle quelques traces, du moins d'elle-même; au lieu que ce dernier moment qui effacera d'un seul trait toute votre vie, s'ira perdre lui-même avec tout le reste dans ce grand gouffre du néant ; il n'y aura plus sur la terre aucuns vestiges de ce que nous sommes. La chair changera de nature ; le corps prendra un autre nom ; "même celui de cadavre ne lui demeurera pas long-temps; il deviendra, dit Tertullien, un je ne sais quoi qui n'a plus de nom dans aucune langue » : tant il est vray que... [...] (Bossuet, $1772: 455)$.

Ce que Urbain et Levesque organisent de la manière suivante :

Il n'y aura plus sur la terre aucuns vestiges de ce que nous sommes : la chair changera de nature ; le corps prendra un autre nom ; " même celui de cadavre ne lui demeurera pas long-temps : il deviendra, dit Tertullien, un je ne sais quoi qui n'a plus de nom dans aucune langue » : tant il est vrai que... [...] (Bossuet, tome 4, 1923 : 268).

La différence entre les deux propositions, à la vérité, n'est pas grande. Toutes deux se heurtent à une commune difficulté : comment construire un ensemble surplombant qui va intégrer les segments ? On comprend que les éditeurs s'y sentent obligés, ayant affaire à un auteur qui passe pour un maître de la " période ". Mais on peut tout de même se poser la question : est-ce bien nécessaire ? Si l'on suit le discours linéairement, en effet, à l'instar de ce que nous faisons lorsque nous entendons un discours par le biais du canal oral, on s'aperçoit que le texte fonctionne très bien sans construction, ni de "phrase ", ni de "période ". Il obéit à une logique qui est généralement celle de la proximité, les segments s'enchaînant les uns aux autres. De ce point de vue, le paramètre interactionnel montre peut-être ici son importance.

La vraie question qui se pose est celle de l'arrêt et de la complétude. Quand ce qui ressemble à un simple déroulement est-il censé s'arrêter ? Ici, on voit bien que c'est le point de vue de la réception qui a conduit les éditeurs à " textualiser " pour ainsi dire le discours, et à le "syntactiser ", en utilisant notamment cet outil fondamental qui est, à l'époque où ils exercent, le point-virgule. Par le point-virgule, notamment, et de façon plus générale tous les signes de ponctuation intermédiaire, on ajoute à la dimension linéaire de la séquentialité une saisie possible par l'ensemble, qui se fait par grands blocs 
saisissables visuellement avant même qu'on n'en entame la lecture. Cette seconde structuration, qui s'appuie également sur la présence des majuscules, crée, soit des phrases, soit des périodes (au sens classiques), les deux manières de voir apparaissant alors comme des effets - grammaticaux et textuels - de la transformation d'un discours produit en un discours lisible.

Examinons pour terminer une lettre manuscrite d'une marchande de cidre normande du milieu du XVIII ${ }^{e}$ siècle.

\subsection{Une lettre peu lettrée du milieu du XVIII ${ }^{\mathrm{e}}$ siècle}

Cette lettre, tirée du Corpus du français familier ancien (cf. Martineau 2015), illustre un autre type encore de réalisation discursive : une production écrite exclusivement destinée à être lue, mais non destinée à être publiée, et émanant d'une personne d'un niveau faible de littéracie.

Monsieur et Cher Couzin

Je suis fachee que Monsieur Domenil na pas zete liure mardy se nest pas mafaute parce que je ne tes pas preuenus le voiturie ariua a deux heure apres midy je ne peu trouer de cheuox ni baril dans lemoment je luis dis qui serait liure dun grand matin il sant feut dun notre cotte qui ne lesse pas de me faire de lapene parce que zauée trouue de cheuox qui fure oblige de sant retourner et je bien eu de lapaine en trouuer cette foies margre toute les paînne que vous zauee eus je ne ces pas si monsieur Domenil saura conten parce que le sidre nest point encorre ennetat de liurer parce quil vient beaucoup de lis Cest pourquoy je ne puis pas vous zen liurer dauantage aprezen il fost pas zen promaitre a personne Car je fait maitre vn mor a la plache du tounos qui est party et je suis si ocupee a deloger et a tou autre afaire que je ne sait au quel antendre cest pourquoy je vous pris de dirre a ceux que vous zennauee promis quil sentroue dun nautre cotte que je nepuis pas leur endonner du tou Je suis bien fachee de ne pas satisfarre à tou cela et je vous suis bien oblige de toute les painne que vous zauée eus pour moy et vous zen remersi bien je suis auec vn profont respec Monsieur et cher Couzin Votres humble et tres obeisante seruante Marguerite lebecq mes compliment a ma Cher Couzine ce quatre decembre 1749

Loin de nous l'objectif de nous livrer ici à une analyse de l'ensemble de ce qui est observable dans le texte, même si on s'en tient aux plans de la syntaxe et de la textualité.

La première chose qu'on remarque naturellement est l'absence totale de ponctuation. En 1749, cette absence ne concerne plus que les manuscrits les moins lettrés. 
La pénétration des habitudes de lecture et des pratiques des imprimeurs commence à être notable chez les plus lettrés (voir Seguin, 2004, sur Saint-Simon, par exemple). Mais cette absence de ponctuation est compensée, pour ce qui est de la segmentation, par le rôle dominant que viennent jouer quelques outils linguistiques grammaticalisés : parce que (présent quatre fois dans les dix premières lignes), bientôt suivi par deux c'est pourquoi. La scriptrice a visiblement son répertoire d'outils qu'elle sur-sollicite, dans des emplois syntaxiques et sémantiques non tout-à-fait homogènes et normés (le parce que de " parce que le sidre nest point encore ennetat de livrer " ne paraissant pas construit de manière habituelle). Le redondance touche d'ailleurs des éléments lexicaux, comme les expressions "je suis fâchée " ou " avoir de la peine ». En fait, c'est le lien entre phraséologie lexicale non grammaticalisée, outils grammaticalisés, et syntaxe, qui paraît modifié de façon à maintenir malgré tout une cohérence et une progression dans la continuité discursive. En effet, les segments sont de nature assez hétérogène, et parfois peu aisés à rattacher les uns aux autres par des outils syntaxiques puissants et clairs (remarquons un "que à tout faire " avant "que je nepuis par leur en donner " un peu avant la fin). C'est pourquoi la dimension de l'énoncé piétine malgré tout dans sa globalité, aucun mode de hiérarchisation n'apparaissant vraiment. Mais ici, ce sont d'autres procédés qui viennent prendre le relais, comme le fort appui sur l'énonciation (présence forte du je) et la dimension de l'interaction (le caractère adressé). Certains outils semi-grammaticalisés sont même employés dans une fonction clairement énonciative (après). Et la fin est marquée par l'apparition plus nette d'un processus d'accumulation de segments (au moyen de deux et très proches l'un de l'autre, notamment).

Au total, on ne peut évidemment pas parler de période dans ce texte. Mais on ne peut pas non plus clairement parler de phrase. Ou plutôt, la notion de phrase ne paraît être le meilleur outil pour aborder le type de segmentation qu'on y observe. En effet, on remarque la grande différence existant entre le bornage à gauche des segments, assez marqué, et le bornage à droite, beaucoup plus indécis. On se trouve face à un effet de début perpétuel. Il se commence plus de phrases qu'il ne s'en termine, pourrait-on dire. On remarquera d'ailleurs que la question de la séquentialité pose presque simultanément la question du début et de la fin des unités. Une ponctuation forte étant tout autant un signe de clôture qu'un signe d'ouverture, la question du " comment commencer" se superpose presque immanquablement à celle du "comment clore ». Et l'activation de ce double programme constitue une contrainte majeure pour le scripteur.

Ainsi, ce type de documents, plus proche du point de vue de la production que ce qui est soumis à publication, où le résultat final est le produit d'une négociation entre point de vue de la production et point de vue de la réception, nous amène à poser une question décisive pour ce qui est la séquentialité : celle-ci se construit-elle majoritairement à partir d'une segmentation consistant à arrêter le déroulé de l'amont, ou à 
partir de la recréation des conditions d'un démarrage ? Dans ce texte, on notera sans peine la présence toujours palpable d'une incomplétude potentielle. On relève d'ailleurs que la lettre se termine de manière commode par une ample formule de salutation qui vient présenter une clôture préformatée à ce qui, sinon, aurait peut-être du mal à s'arrêter.

\section{Conclusion}

Dans cet article, nous avons essayé de formuler quelques-unes des questions qui peuvent se poser lorsqu'on examine en diachronie les phénomènes de la segmentation et de la séquentialité parfois décrits en termes de "phrase " et de "période ». Nous avons d'abord posé la question de savoir s'il fallait plutôt partir d'un modèle ou plutôt partir des usages, de l'observable. Comme on l'aura compris, sans sous-estimer les capacités des modèles, on considère que du fait de son appartenance partielle à la sphère du discours, la problématique a beaucoup à nous apprendre si on repart des usages. Nous avons ensuite passé en revue un certain nombre de paramètres qui me semblent nécessaires à prendre en compte si l'on veut suivre la problématique dans la diachronie : paramètres de textualité, du genre, de la littéracie, des cadres de pensée, du caractère plus ou moins adressé des discours, de la médialité. À travers l'examen rapide de trois textes des XVII ${ }^{e}$ et XVIII ${ }^{e}$ siècles, nous avons ensuite cherché à montrer que, si l'on part d'un examen au plus près des usages de la question de la séquentialité, la " phrase " n'apparaissait, ni comme un donné d'avance du point de vue de la production, ni comme une obligation de lecture du point de la réception. Bien plutôt, elle apparaît comme une hypothèse, une potentialité discursive, une unité de passage, qui se construit dans l'acte même d'énonciation, et se déduit de certaines conditions de réception, et à laquelle on peut ne pas donner les mêmes contours selon qu'on la produit ou qu'on la reçoit.

La question de la séquentialité semble également demander qu'on donne un sens particulier à ce qu'on peut appeler "diachronie ". Bon nombre des paramètres à prendre en compte sont en effet plutôt du ressort de l' " histoire " que de la diachronie, et même plutôt de ce qu'on appelle ordinairement « histoire externe ». Les manières de comprendre les questions de segmentation et de séquentialité ont connu dans l'histoire des modulations qui sont liées à l'historicité des pratiques et des cadres de pensée.

De ce point de vue, on pourra considérer que, entre les XVII ${ }^{e}$ et XVIII ${ }^{e}$ siècles, la segmentation en ce que nous appelons aujourd'hui "phrases" gagne indiscutablement du terrain. Cette segmentation l'emportera dans les usages lettrés à partir de la fin du XVIII ${ }^{e}$ siècle, le mode plus souple en association libre de segments restant très présent dans les textes des peu lettrés, avant de reparaître dans des expérimentations littéraires des $\mathrm{XIX}^{\mathrm{e}}$ et $\mathrm{XX}^{\mathrm{e}}$ siècles, et aujourd'hui dans l'écrit numérique. D'une certaine manière, on observe ainsi une étrange persistance conjointe, à la fois de la phrase et de 
la non-phrase. Ce qu'on peut appeler "phrase " nous dit toujours aussi quelque chose sur ce qu'elle n'est pas, et nous le rend sensible, parfois jusqu'à le formuler.

\section{RÉFÉRENCES BIBLIOGRAPHIQUES}

a) Sources primaires

ANON (1860): Nouveau manuel des Salles d'Asile. Paris, Dezobry.

BOSQUET, Jean (2005 [1586]) : Elemens ou institutions de la langue françoise..., éd. de C. Demaizière. Paris, Champion.

BossueT, Jacques Bénigne, Manuscrits, XI-XV Sermons. XII Sermons pour le temps du Jubilé, pour le vendredi et le samedi après les Cendres, sermons du Carême jusqu'à la fin de la quatrième semaine. Manuscrit $\mathrm{BnF}$, fr. 12822. Gallica. URL: https://gallica.bnf.fr/ark:/12148/btv1b53000317w/f1.item.r=fran\%C3\%A7ais\%201 2822

Bossuet, Jacques Bénigne (1772) : CEuvres de Messire Jacques Bénigne Bossuet. Éd. de René Déforis. Paris, Boudet.

BOSSUET, Jacques Bénigne (1914-1921) : Euvres oratoires, éd. critique de J. Lebarcq revue par Charles Urbain et Eugène Levesque. Paris, Hachette et Desclées de Brouwer, 4 vols.

BUFFIER, Claude (1709) : Grammaire françoise sur un plan nouveau. Paris, N. Le Clerc.

MARTINEAU, France (2015): Projet Corpus du français familier ancien, http://polyphonies.uottawa.ca/fr/corpus/i-corpus-de-francais-familier-ancien/.

SOREL, Charles (1688) : La Science universelle, tome IV. Paris, Jean Guignard.

TRISTAN L'HERMITE [François l'Hermite] (1642) : Lettres meslées du sieur de Tristan. Paris, A. Courbé. [Reproduction en ligne par "Google Recherche de Livres", URL: https://www.google.com/search?tbm=bks\&q=lettres+meslees]

VAUGELAS, Claude Favre de (2009 [1647]) : Remarques sur la langue françoise, éd. Z. Marzys. Genève, Droz.

\section{b) Études}

ADAM, Jean-Michel (2003) : «Entre la phrase et le texte. La période et la séquence comme niveaux intermédiaires de cohésion ". Québec français, 128, 51-54.

Apothéloz, Denis ; Anne Grobet \& Simona PeKareK DoeHler (2007) : «Séquentialité et temporalité du discours ». Cahiers de praxématique, 48, 23-56.

ARABYAN, Marc (2018) : " Histoire et emplois de l'alinéa ouvrant en diachronie (XIII ${ }^{e}-X{ }^{2} I^{e}$ siècles) ». Signata, 9. DOI : https://doi.org/10.4000/signata.1814

BADIOU-MONFERRAN, Claire (2000): Les conjonctions de coordination ou «l'art de lier ses pensées » chez La Bruyère. Paris, Champion.

BADIOU-MONFERRAN Claire \& Daniela CAPIN (2020) : "Comment identifier le et" de relance " en diachronie longue ? ", in Marta Saiz-Sánchez, Amalia Rodríguez Somolinos, \& Sonia Gómez-Jordana Ferary, (éds), Marques d'oralité et représentation de l'oral en français, Chambéry, Presses Universitaires Savoie Mont Blanc (Collection Langages, no 20), 369-390. 
BAUDRY, Janine \& Philippe CARON [éds] (1998) : Problèmes de cohésion syntaxique de 1550 à 1720. Limoges, PULIM.

BERRENDONNER, Alain (2017) : «La notion de période (note terminologique) ». Encyclopédie grammaticale du français. URL : http://encyclogram.fr

BENZITOUN, Christophe \& Frédéric SABIO (2010) : «Où finit la phrase ? Où commence le texte? ». Discours, 7. URL : http://discours.revues.org/7966

Blanche-Benveniste, Claire (1997) : "The Units of Written and Oral Language », in Clotilde Pontecorvo (éd.), Writing Development. An Interdisciplinary view, Amsterdam, John Benjamins, 21-46.

BRUHN, Jorgen (2016) : "What is Mediality and (How) does it matter ? Theoretical Terms and Methodology ». The Intermediality of Narrative Literature. Londres, Palgrave Mac Millan, 13-40.

CHANQUOY, Lucile \& Michel FAYOL (1995) : «Analyse de l'évolution et de l'utilisation de la ponctuation et des connecteurs, dans deux types de texte. Étude longitudinale du CP au CE2 ». Enfance, 48 : 2, 227-241.

Charolles, Michel ; Pierre Le GoffiC \& Mary-Annick MORel [éds] (2002) : Verbum, 24 : 1-2 [Y a-t-il une syntaxe au-delà de la phrase ?].

CHERVEL, André (2008) : Histoire de l'enseignement du XVII au XXe siècle. Paris, Retz.

Colombat, Bernard ; Bernard COMBeTtes, Valérie Raby \& Gilles SiOuffi [éds] (2018) : Histoire des langues et histoire des représentations des langues. Paris, Champion.

COMBeTtes, Bernard (1983) : Pour une grammaire textuelle. Bruxelles / Paris, De Boeck / Duculot.

COMBETTES, Bernard [éd.] (2003) : Évolution et variation en français préclassique, Études de syntaxe. Paris, Honoré Champion.

DAUVOIS, Nathalie \& Jacques DÜRRENMATT [éds] (2011) : La ponctuation à la Renaissance. Paris, Garnier.

DÜRRENMATT Jacques (2011) : "Grandeur et décadence du point-virgule ». Langue française, $172,32-42$.

ELALOUF, Marie-Laure (2014) : "La notion de phrase de base dans la pratique des enseignants français : choix terminologiques et enjeux théoriques ». Repères, 49, 33-55.

ERNST, Gerhardt (2003) : "Les peu lettrés devant les normes de la textualité », in Dietmar Osthus, Claudia Polzin-Haumann \& Christian Schmitt (éds), La norme linguistique. Théorie - pratique - médias - enseignement. Bonn, Romanistischer Verlag Hillen, 8398.

GAUTIER, Antoine (2006) : Unité et discontinuité : une approche systématique et épistémologique de la phrase. Thèse en Sciences du langage sotenue à ñ'Université Paris-Sorbonne sous la direction d'Olivier Soutet.

GAUTIER, Antoine (2010) : «Syntaxe et ponctuation en conflit. Le point est-il une limite de la rection ?". Travaux de linguistique, 60, 91-107. 
Groupe De Fribourg (2012) : Grammaire de la période. Berne, Peter Lang.

Guillaume, Gustave (2005) : Leçons de linguistique, tome 17. Québec / Lille, Presses de l'Université Laval / Klincksieck.

HiRSCH, Fabrice \& Christelle DODANE [éds] (2018) : Langue française, 211 [Organisation spatiale et temporelle des pauses en parole et en discours].

KAVANAGH, Eric ; Jacynthe ROBERGE \& Isabelle SPERANO (2016) : "Typologie exploratoire des affordances textuelles ». Pratiques, 171-172. DOI : https://doi.org/10.4000/pratiques.3187

LACHERET, Anne \& Bernard VICTORRI (2002) : « La période intonative comme unité d'analyse pour l'étude du français parlé : modélisation prosodique et enjeux linguistiques ». Verbum, 24 : 1-2 [Ya-t-il une syntaxe au-delà de la phrase ?], 55-72.

LECOINTE, Jean (2002) : «L'organisation périodique du style coupé dans le livre III des Essais ». Styles, genres, auteurs, 2, 9-24.

LLAMAS-POMBO, Elena (2008) : «Ponctuer, éditer, lire. État des études sur la ponctuation dans le livre manuscrit ". Syntagma, Revista del Instituto de Historia del Libro y de la Lectura, 2, 129-171.

LLAMAS-POMBO, Elena (2017) : «Graphie et ponctuation du français médiéval. Système et variation ", in Gabriella Parussa, Maria Colombo Timelli \& Elena Llamas-Pombo (éds), Enregistrer la parole, écrire la langue dans la diachronie du français. Tübingen, Günter Narr, 39-88.

LAVRENTIEV, Alexei (2009): Tendances de la ponctuation dans les manuscrits et incunables français en prose, $d u X I I I^{e}$ au $X V^{e}$ siècle. Thèse de doctorat en sciences du langage sous la direction de Christiane Marchello-Nizia. Lyon, ENS-LSH. URL : http://tel.archivesouvertes.fr/tel-00494914

LE GOFFIC, Pierre (2008) : "Phrase, séquence, période ", in Dan Van Raemdonck (éd.), Modèles syntaxiques. Bern, Peter Lang, 329-356.

LE GOFFIC, Pierre (2011) : «Phrase et intégration textuelle ». Langue française, $170: 2,11-28$.

MinistÈRE DE L'ÉDUCATION NATIONALE (2020) : Grammaire du français. Terminologie grammaticale, réalisé sous la direction de Philippe Monneret et Fabrice Poli. URL: https://eduscol.education.fr/

MOREL, Mary-Annick \& Laurent DANON-BOILEAU (1998): Grammaire de lintonation: l'exemple du français. Paris, Ophrys.

PRAT, Marie-Hélène \& Fabienne BOISSIÉRAS [éds] (2012) : Hiérarchisation, énonciation. Leuven, Peeters.

RABY, Valérie (2016) : " Ponctuation et invention de la phrase complexe chez les grammairiens du XVIII ${ }^{e}$ siècle », in Sabine Pétillon, Fanny Rinck \& Antoine Gautier (éds), La Ponctuation à l'aube du XXI siècle : perspectives historiques et usages contemporains. Limoges, Éditions Lambert-Lucas, 63-78.

RABY, Valérie (2018) : Les théories de l'énoncé dans la grammaire générale. Lyon, ENS Éditions. 
RANNOUX, Catherine \& Jacques DÜRRENMATT [éds] (1997) : La phrase. Mélanges offerts à Jean-Pierre Seguin. Poitiers, La Licorne.

RoubaUd, Marie-Noëlle (2016) : "La phrase à l'épreuve des textes scolaires (élèves de 5 à 8 ans)». Lidil, 54, 93-114.

SEGUIN, Jean-Pierre (1993) : L'Invention de la phrase au XVIII siècle. Contribution à l'histoire du segment linguistique en français. Louvain / Paris, Peeters, Bibliothèque de l'Information Grammaticale.

SEGUIN, Jean-Pierre (2004) : "Sens de la phrase et ponctuation chez un auteur du XVIII ${ }^{e}$ siècle. L'exemple de Saint-Simon ». L'Information Grammaticale, 101, 9-13.

SEGUIN, Jean-Pierre (2013) : "Problèmes de définition du style coupé au XVIII ${ }^{e}$ siècle ». Cahiers Forell 1, URL : https://cahiersforell.edel.univ-poitiers.fr/index.php?id=94

SERÇA, Isabelle (2012) : Esthétique de la ponctuation. Paris, Gallimard.

SiOUfFI, Gilles (2019a) : «La phrase dans les Lettres meslées de Tristan L'Hermite ». Cahiers Tristan L'Hermite, XLI, 77-93.

SioufFI, Gilles [éd.] (2019b) : Phrase et période entre les XVI et XVIII siècles. Dossier de Verbum, $41: 2$.

SIOUFFI, Gilles (2019c) : "Médialité et séquentialité ». Cahiers de praxématique, 71, URL: http://journals.openedition.org/praxematique/4962

SIOUFFI, Gilles [dir.] (2020) : Une histoire de la phrase française. Des Serments de Strasbourg aux écritures numériques. Arles, Actes Sud.

STEUCKARDT, Agnès (2015) : "Sans point ni virgule », in Agnès Steuckardt (dir.), Entre village et tranchées. L'écriture de poilus ordinaires. Uzès, Inclinaison, 91-101.

WEINRICH, Heinrich (1989) : Grammaire textuelle du français. Paris, Didier. 